\title{
Evaluación del densificado de la madera de Gyrocarpus americanus Jacq. en su densidad y módulo de elasticidad
}

\author{
Evaluation of densification of Gyrocarpus americanus Jacq. in its density and modulus \\ of elasticity
}

\section{Avaliação da densificação de Gyrocarpus americanus Jacq. em sua densidade e módulo de elasticidade}

Javier Ramón Sotomayor-Castellanos ${ }^{1}$

Recibido: diciembre de 2017

Aceptado: abril de 2018

Para citar este artículo: Sotomayor-Castellanos, J. R. (2018). Evaluación del densificado de la madera de Gyrocarpus americanus Jacq. en su densidad y módulo de elasticidad. Revista Científica, 32(2), 157-168. Doi: https:// doi.org/10.14483/23448350.128330

\section{Resumen}

El densificado de la madera es un mecanismo de deformación que se presenta durante la manufactura de productos compuestos. Esta tecnología pretende la estabilización dimensional de la madera e incrementar sus propiedades mecánicas. El objetivo de la investigación fue densificar madera de Gyrocarpus americanus Jacq., evaluar el efecto del tratamiento en la densidad y en el módulo de elasticidad. Inicialmente, la madera se hidrató en agua, durante 72 horas y con temperatura de $20{ }^{\circ} \mathrm{C}$. Posteriormente, las probetas se suavizaron durante 4 horas en agua caliente con temperatura de $93.5^{\circ} \mathrm{C}$. A continuación, se prensaron las probetas con un dispositivo fabricado especialmente para la investigación. La estrategia de investigación consistió en determinar la densidad y realizar pruebas de flexión estática, antes y después del tratamiento. Los coeficientes de compresión y de densificado fueron evaluados después del tratamiento. Se prepararon 32 probetas normalizadas y se realizaron pruebas de flexión estática, antes y después del densificado. Para estos resultados se realizaron pruebas de diferencias de medias para muestras independientes, y se calcularon las regresiones entre los valores del módulo de elasticidad como variable dependiente en función de la variable independiente densidad. El coeficiente de compresión fue de $56.3 \%$ y el coeficiente de densificado fue de $50.3 \%$. Después del tratamiento la densidad de Gyrocarpus americanus Jacq. aumentó $102 \%$ y su módulo de elasticidad se incrementó en $113 \%$. Los índices de densificado, elástico y de calidad confirmaron que el tratamiento de densificado higro-termo-mecánico produjo buenos resultados para madera de baja densidad.

Palabras clave: densidad de la madera, índice de calidad, índice de densificado, índice elástico, módulo de elasticidad. 


\section{Abstract}

The densified of wood is a mechanism of deformation that occurs during the manufacture of composite products. This technology aims at the dimensional stabilization of wood and increase its mechanical properties. The objective of the research was to densify Gyrocarpus americanus Jacq. wood and to evaluate the effect of the treatment on density and modulus of elasticity. Initially, the wood was hydrated in water for $72 \mathrm{~h}$ and at a temperature of $20^{\circ} \mathrm{C}$. Subsequently, the specimens were softened for $4 \mathrm{~h}$ in hot water at a temperature of $93.5^{\circ} \mathrm{C}$. The specimens were then pressed with a device made especially for the investigation. The research strategy was to determine density and perform static flexion tests before and after treatment. The compression and densification coefficients were evaluated after treatment. Thirty-two standardized specimens were prepared and static flexural tests were performed before and after densification. For the before and after densified results tests of mean differences for independent samples were performed. The regressions between the values of the modulus of elasticity as a dependent variable were calculated as a function of the independent variable density. The compression coefficient was $56.3 \%$ and the densification coefficient was $50.3 \%$. After treatment, the density of G. americanus increased $102 \%$ and its modulus of elasticity increased by $113 \%$. The densified, elastic and quality indices confirmed that the densified hygrothermo-mechanical treatment produced good results for low-density wood.

Keywords: wood density, quality index, densification index, elastic index, modulus of elasticity.

\section{Resumo}

A densificação da madeira é um mecanismo de deformação que ocorre durante o fabrico de produtos compostos. Esta tecnologia visa a estabilização dimensional da madeira e aumenta suas propriedades mecânicas. O objetivo da pesquisa foi densificar a madeira de Gyrocarpus americanus Jacq. e avaliar o efeito do tratamento na densidade e módulo de elasticidade. Inicialmente, a madeira foi hidratada em água, por $72 \mathrm{~h}$ e a uma temperatura de $20^{\circ} \mathrm{C}$. Posteriormente, os espécimes foram suavizados durante $4 \mathrm{~h}$ em água quente a uma temperatura de $93.5^{\circ} \mathrm{C}$. Em seguida, os espécimes foram pressionados com um dispositivo especialmente fabricado para pesquisa. A estratégia de pesquisa foi determinar a densidade e realizar testes de flexão estática antes e após o tratamento. Os coeficientes de compressão e densificação foram avaliados após o tratamento. Foram preparados 32 espécimes padronizados e realizaram-se testes de flexão estática antes e após a densificação. Para os resultados antes e depois da densificação, as diferenças médias foram testadas para amostras independentes. As regressões foram calculadas entre os valores do módulo de elasticidade como variável dependente em função da densidade variável independente. O coeficiente de compressão foi de $56.3 \%$ e o coeficiente de densificação foi de $50.3 \%$. Após o tratamento, a densidade de G. americanus aumentou $102 \%$ e seu módulo de elasticidade aumentou em $113 \%$. Os índices densificados, elásticos e de qualidade confirmaram que o tratamento de densidades higro-termo-mecânicas produziu bons resultados para madeira de baixa densidade.

Palavras-chaves: densidade da madeira, índice de qualidade, índice de densificação, índice de elasticidade, módulo de elasticidade.

\section{Introducción}

La llegada al mercado de madera aserrada de especies de baja densidad, que proviene de plantaciones forestales, así como la incorporación en usos estructurales de maderas de especies de nuevo aprovechamiento, ha suscitado la caracterización de las propiedades mecánicas de especies susceptibles de aumentar artificialmente su densidad (Yoshihara y Tsunematsu, 2007).

El densificado de la madera es un importante mecanismo de deformación que se presenta en varios procesos de manufactura de productos de madera reconstituida y de componentes estructurales. Este tratamiento involucra la compresión transversal de la madera e implica su comportamiento elasto-plástico al incluir grandes deformaciones (Aimene y Nairn, 2015).

Los tratamientos de densificado de la madera, de tipo higro-termo-mecánico, son de carácter 
eco-sustentable y pueden ser clasificados desde tres perspectivas: el que tiene por objeto el plastificado de la madera, el que pretende la estabilización dimensional de la madera y el que tiene por propósito incrementar sus propiedades mecánicas. Para lograr estos objetivos se han experimentado diferentes procedimientos de densificado y condiciones de ensayo (Asaba y Nishimura, 2001; Hayashi y Nishimura, 2001; Kubojima, Ohtani y Yoshihara, 2004; Haller y Wehsener, 2004; Tang, Nakao y Zhao, 2004; Navi y Pizzi, 2015).

En el densificado de la madera, se emplean tratamientos de impregnación (Hill, 2006) o de compresión (Navi y Girardet, 2000), se incrementan sus propiedades de resistencia mecánica (Kutnar, Kamke y Sernek, 2008), se mejoran sus características higroscópicas (Skyba, Schwarze y Niemz, 2009; Fang, Cloutier, Blanchet, Koubaa y Mariotti, 2011) y se mejora su resistencia al deterioro ocasionado por agentes biológicos (Fang, Mariotti, Cloutier, Koubaa y Blanchet, 2012b). Así, el incremento artificial de la densidad de la madera, puede diversificar sus usos potenciales (Blomberg, Persson y Blomberg, 2005). Por ejemplo: los pliegos o láminas de madera densificada pueden ser incorporados en la fabricación de madera laminada los cuales pueden ser empleados para la fabricación de muebles, de pisos y como componente constructivo en edificaciones (Eckelman, 1993; Hayashi y Oshiumi, 1993; Ozarska, 1999; Erdil, Kasal, Zhang, Efe y Dizel, 2009).

La intensidad del tratamiento de densificado es evaluada con dos parámetros: el coeficiente de compresión (Sandberg y Navi, 2007), el cual explica el grado en que la pieza de madera es deformada en una dirección comparativamente a su tamaño original; y el coeficiente de densificado (Blomberg et al., 2005), que representa el nivel de compactación del volumen respecto a la masa de la pieza en cuestión. La calidad del tratamiento de densificado es valorada con el índice de densificado y con el índice elástico, ambos necesarios para calcular el índice de calidad del densificado (Blomberg et al., 2005). El índice de densificado expresa el aumento en la densidad de la pieza de madera en tratamiento. Por su parte, el índice elástico explica la ganancia en el valor del módulo de elasticidad de la madera densificada en comparación con madera sin tratamiento. Ambos indicadores se combinan en el índice de calidad del densificado para explicar la relación entre la ganancia en densidad en relación al incremento en el módulo de elasticidad.

Todo proyecto ingenieril con madera requiere conocer el módulo de elasticidad de la especie que se va a emplear. De tal forma que la densidad de la madera es el parámetro de referencia, en su clasificación como material de ingeniería y como la característica física, para predecir el módulo de elasticidad (Niklas y Spatz, 2010). De igual manera, el módulo de elasticidad es un criterio de selección para el cálculo de productos y elementos estructurales de madera (Dinwoodie, 2000).

En México, la especie G. americanus. se distribuye en el estado de Oaxaca, y su distribución se extiende por Centroamérica, Colombia y norte de Venezuela (Rivera, 2006). Su poca resistencia y durabilidad la hacen inadecuada para usos muy finos y es más apropiada para cajas de embalaje, cubiertas para protección, soportes para muebles y posiblemente la extracción de pulpa (Cordero y Boshier, 2003).

Sus características físicas y mecánicas están reportadas por Sotomayor (2015). En la revisión de bibliografía, no se detectó información sobre el efecto del densificado de la madera de G. americanus en su módulo de elasticidad evaluado en flexión estática. Debido a su baja densidad, que, según el trabajo de Sotomayor, fluctúa entre 350 y $410 \mathrm{~kg} \mathrm{~m}^{-3}$, no es atractiva para uso estructural. No obstante, si se aumenta de manera artificial su densidad, posiblemente la madera de G. americanus presentará potencial para la fabricación de productos de valor agregado.

El objetivo de la investigación fue densificar madera de G. americanus con un tratamiento higro-termo-mecánico y evaluar su efecto en la densidad y en el módulo de elasticidad. 


\section{Metodología}

El material experimental utilizado y el procedimiento de densificado aplicado a la madera son los mismos empleados por Sotomayor (2016), mas no la configuración de las pruebas mecánicas. Mientras que Sotomayor realizó pruebas de ondas de esfuerzo y determinó el módulo dinámico en la dirección longitudinal de la madera, en la presente investigación se realizaron pruebas de flexión transversal y se determinó el módulo de elasticidad estático. Asimismo, los índices de densificado y de calidad del densificado para G. americanus están reportados en Sotomayor (2017).

Se recolectaron piezas aserradas de madera de G. americanus Jacq., en el estado de Michoacán, México. La especie se identificó en el Laboratorio de Mecánica de la Madera, de la Facultad de Ingeniería en tecnología de la Madera, de la Universidad Michoacana de San Nicolás de Hidalgo. Se prepararon 32 probetas con sección de 0.02 $\mathrm{m} \times 0.02 \mathrm{~m}$ de sección transversal y de $0.32 \mathrm{~m}$ de longitud adaptando la norma ISO 3129:2012 (International Organization for Standardization, 2012). Las probetas fueron orientadas en las siguientes direcciones de la madera: radial, tangencial y longitudinal. Las probetas estuvieron libres de irregularidades de crecimiento tales como nudos y desviación de la fibra. La madera se acondicionó durante 24 meses en una cámara con una temperatura de $20^{\circ} \mathrm{C}\left( \pm 1{ }^{\circ} \mathrm{C}\right)$ y una humedad relativa del aire de $65 \%( \pm 2 \%)$ hasta que alcanzó un peso constante.

La estrategia de investigación consistió en determinar la densidad y el contenido de humedad de la madera y realizar pruebas de flexión estática antes y después del tratamiento de densificado. Los coeficientes de compresión y de densificado fueron evaluados después del tratamiento.

El contenido de humedad $(\mathrm{CH})$ se determinó con un grupo complementario de probetas con dimensiones de $0.02 \mathrm{~m} \times 0.02 \mathrm{~m} \times 0.32 \mathrm{~m}$, en las direcciones radial, tangencial y longitudinal respectivamente adaptando la norma ISO
13061-2:2014. La determinación de la densidad al momento del ensayo $\left(\rho_{\mathrm{CH}}\right)$ se calculó con la relación entre el peso de la probeta al momento de las pruebas y su volumen correspondiente.

El tratamiento de densificado de madera siguió el procedimiento utilizado por Sotomayor (2016) y consistió en los pasos siguientes:

1. Hidratado de la madera, a temperatura de 20 ${ }^{\circ} \mathrm{C}$, durante 72 horas.

2. Suavizado en agua con una temperatura de $93.5^{\circ} \mathrm{C}$, durante 4 horas.

3. Prensado mecánico de las probetas en una maquina universal de pruebas mecánicas Tinius-Olsen ${ }^{\circledR}$ con capacidad de 300 kN. La velocidad de desplazamiento de la carga fue de $1 \mathrm{~mm} \mathrm{~min}{ }^{-1}$. El tiempo de cerrado del dispositivo de densificado fue de $15 \mathrm{~min}$. La operación terminó cuando se alcanzó la taza de compresión objetivo de 56\%, con una carga promedio de $120 \mathrm{kN}$.

4. Secado al interior de un horno con una temperatura de $80^{\circ} \mathrm{C}$, durante 24 horas.

5. Estabilizado en una cámara de acondicionamiento con una temperatura de $20{ }^{\circ} \mathrm{C}$ y con una humedad relativa del aire de $65 \%$, durante 60 días.

El coeficiente de compresión se calculó con la fórmula (Sandberg y Navi, 2007):

$$
\varphi_{\mathrm{c}}=\left(\frac{\mathrm{R}_{\mathrm{ad}}-\mathrm{R}_{\mathrm{dd}}}{\mathrm{R}_{\mathrm{ad}}}\right) \times 100
$$

Donde:

$\varphi_{\mathrm{c}}=$ coeficiente de compresión (\%)

$\mathrm{R}_{\mathrm{ad}}=$ espesor de la probeta antes del densificado $(\mathrm{m})$

$R_{d d}=$ espesor de la probeta después del densificado $(\mathrm{m})$

El coeficiente de densificado se calculó con la fórmula (Blomberg et al., 2005): 
$\lambda_{\rho}=\left(\frac{\rho_{\mathrm{dd}}-\rho_{\mathrm{ad}}}{\rho_{\mathrm{dd}}}\right) \times 100$

Donde:

$\lambda_{\rho}=$ coeficiente de densificado $(\%)$

$\rho_{\mathrm{ad}}=$ densidad de la probeta antes del densificado $(\mathrm{m})$

$\rho_{\mathrm{dd}}=$ densidad de la probeta después del densificado $(\mathrm{m})$

Las pruebas de flexión estática antes del densificado se realizaron en una maquina universal de pruebas mecánicas Tinius-Olsen $®$ con capacidad de 300 kN, en el Laboratorio de Mecánica de la Madera, de la Facultad de Ingeniería en Tecnología de la Madera, de la Universidad Michoacana de San Nicolás de Hidalgo, en Morelia, Michoacán, México, de acuerdo al protocolo propuesto por Sotomayor, Ramírez y Olguín (2014). Las pruebas de flexión estática después del densificado se realizaron siguiendo el mismo protocolo, pero en una maquina universal de pruebas mecánicas Sintech 30/D testing machine ${ }^{\circledR}$ con capacidad de 600 kN, en el Departamento de Ciencias de la Madera, de la Universidad de Columbia Británica, Canadá.

El módulo de elasticidad se calculó con la fórmula (Koman, Feher, Abraham y Taschner 2013):

$$
\mathrm{MOE}=\frac{\mathrm{PL}^{3}}{48 \mathrm{y} \mathrm{I}}
$$

Donde:

MOE $=$ módulo de elasticidad $\left(\mathrm{N} \mathrm{m}^{-2}\right)$

$\mathrm{P}=\operatorname{carga}(\mathrm{N})$

$y=$ Deflexión $(m)$

$\mathrm{L}=$ distancia entre apoyos $(\mathrm{m})$

$\mathrm{I}=$ momento de inercia $\left(\mathrm{m}^{4}\right)$

Para fines de análisis de la calidad del tratamiento, el índice de densificado fue calculado con la fórmula (Blomberg et al., 2005):
$\mathrm{I}_{\rho}=\frac{\rho_{\mathrm{dd}}}{\rho_{\mathrm{ad}}}$

Donde:

$\mathrm{I}_{\rho}=$ índice de densificado

$\rho_{\mathrm{dd}}=$ densidad después del densificado $\left(\mathrm{kg} \mathrm{m}^{-3}\right)$

$\rho_{\text {ad }}=$ densidad antes del densificado $\left(\mathrm{kg} \mathrm{m}^{-3}\right)$

El índice elástico fue calculado con la fórmula (Blomberg et al., 2005):

$$
I_{M O E}=\frac{M_{d d}}{M O E_{a d}}
$$

Donde:

$\mathrm{I}_{\mathrm{MOE}}=$ índice elástico

$\mathrm{MOE}_{\mathrm{dd}}=$ módulo de elasticidad después del densificado $\left(\mathrm{N} \mathrm{m}^{-2}\right)$

$\mathrm{MOE}_{\mathrm{ad}}=$ módulo de elasticidad antes del densificado $\left(\mathrm{N} \mathrm{m}^{-2}\right)$

El índice de calidad del densificado fue calculado con la fórmula (Blomberg et al. 2005):

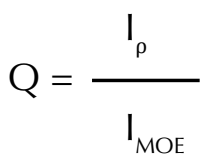

Donde:

$\mathrm{Q}=$ índice de calidad del densificado

$I_{\rho}=$ índice de densificado

$\mathrm{I}_{\mathrm{MOE}}=$ índice elástico

\section{Diseño experimental}

Para las variables densidad, módulo de elasticidad, coeficiente de compresión, coeficiente de densificado, índice de densificado, índice elástico e índice de calidad, se determinaron las estadísticas: media aritmética, desviación estándar y 
coeficiente de variación. Se realizaron pruebas de asimetría y curtosis para verificar la normalidad de las muestras.

Una vez que se verificó que las muestras provenían de distribuciones normales, se diseñó un experimento siguiendo las recomendaciones de Gutiérrez y de la Vara (2011). Las variables de respuesta fueron la densidad de la madera y el módulo de elasticidad. Los índices relacionados con la intensidad y la calidad del tratamiento se analizaron como variables derivadas. El tratamiento de densificado se consideró el factor de variación. El experimento consistió en pruebas $t$ de Student para muestras pareadas de diferencias de medias para un nivel de confianza de 95\%. La hipótesis nula $H_{0}: x_{-} 1-x_{-} 2=0$ se contrastó con la hipótesis alterna $\mathrm{H}_{\mathrm{A}}$ : $\mathrm{x}_{-} 1-\mathrm{x}_{-} 2 \neq 0$. Los subíndices 1 y 2 representan el valor de las variables de respuesta para cada uno de los estados antes del densificado y después del densificado.

Para los resultados antes y después del densificado se calcularon las regresiones y sus coeficientes de determinación entre los valores del módulo de elasticidad como variable dependiente en función de la densidad como variable independiente.

\section{Resultados}

La densidad, el módulo de elasticidad, el coeficiente de compresión y el coeficiente de densificado de madera de G. americanus se presentan en la tabla 1.

Los valores de asimetría y de curtosis de las pruebas de normalidad para la densidad de la madera (Asimetría $=1.77$ y curtosis=0.24) y para el módulo de elasticidad (Asimetría=-0.83 y curtosis=1.96) de G. americanus se situaron en el intervalo $-2 y+2$. De tal forma que se admite que la muestras provienen de distribuciones normales; lo que permitió realizar las pruebas de diferencia de medias. Las pruebas $t$ de Student para la densidad de la madera y para el módulo de elasticidad indicaron valores de $P<0.0001(\alpha=0.05)$. Estos resultados confirman que existe una diferencia significativa entre los valores antes y después del densificado de las variables de respuesta de la madera de G. americanus. De aquí se deriva que el

Tabla 1. Densidad, módulo de elasticidad, coeficiente de compresión y coeficiente de densificado para madera de G. americanus.

\begin{tabular}{|c|c|c|c|c|}
\hline \multicolumn{5}{|c|}{ Antes del densificado } \\
\hline & $\rho_{\mathrm{CH}}$ & MOE & & \\
\hline & $\left(\mathrm{kg} \mathrm{m}^{-3}\right)$ & $\left(\mathrm{MN} \mathrm{m}^{-2}\right)$ & - & - \\
\hline $\bar{x}$ & 397 & 6157 & - & - \\
\hline$\sigma$ & 28 & 1030 & - & - \\
\hline $\mathrm{CV}$ & 7,0 & 16,7 & - & - \\
\hline \multicolumn{5}{|c|}{ Después del densificado } \\
\hline & $\begin{array}{c}\rho_{\mathrm{CH}} \\
\left(\mathrm{kg} \mathrm{m}^{-3}\right)\end{array}$ & $\begin{array}{c}\mathrm{MOE} \\
\left(\mathrm{MN} \mathrm{m}^{-2}\right)\end{array}$ & $\begin{array}{r}\varphi_{c} \\
(\%)\end{array}$ & $\begin{array}{r}\lambda_{\rho} \\
(\%)\end{array}$ \\
\hline $\bar{x}$ & 800 & 13123 & 56.3 & 50.3 \\
\hline$\sigma$ & 73 & 2026 & 0.60 & 1.94 \\
\hline CV & 9.2 & 15.4 & 1.1 & 3.9 \\
\hline
\end{tabular}

$\rho_{\mathrm{CH}}=$ densidad; $\mathrm{MOE}=$ módulo de elasticidad; $\varphi_{\mathrm{c}}=$ coeficiente de compresión; $\lambda_{\rho}=$ coeficiente de densificado; $\overline{\mathrm{x}}=$ media; $\sigma=$ desviación estándar; $C V=$ coeficiente de variación en porciento.

Fuente: elaboración propia del autor. 
tratamiento de densificado modificó la densidad y el módulo de elasticidad.

La magnitud del contenido de humedad de la madera de G. americanus antes del densificado fue de $12.2 \%$ y después del densificado disminuyó a $11.91 \%$, lo que representa una variación de $2.5 \%$. La variación del módulo de elasticidad de la madera atribuido a la modificación en su contenido de humedad, después del densificado, puede ser estimada empleando el coeficiente de higroelasticidad reportado por Sotomayor y Villaseñor (2016), para madera de Pinus douglasiana con densidad $\rho_{C H}=521 \mathrm{~kg} \mathrm{~m}^{-3}$, el cual es de $200 \mathrm{MN} \mathrm{m}^{-2} \%{ }^{-1}$. Así que la diferencia en el módulo de elasticidad sería de $498 \mathrm{MN} \mathrm{m}^{-2}$, lo que representa un porcentaje de $0.92 \%$ del módulo de elasticidad antes del densificado. De tal forma, se consideró que el contenido de humedad en la madera no influyó en los resultados.

El coeficiente de densificado fue $10.7 \%$ menor que el coeficiente de compresión (tabla 1). Esta diferencia puede ser explicada por el hecho de que el prensado mecánico de la probeta se realizó en la dirección radial de la madera y el dispositivo de densificado no bloqueó las caras laterales de la probeta. Esto permitió la deformación de la madera en la dirección tangencial, es decir, transversal a la dirección de la carga. De tal forma, que la compactación del volumen en esta dirección no se efectuó de manera uniforme.

Por otra parte, la madera de G. americanus clasifica como una madera con un porcentaje de espacios vacíos muy alto (77.1\%) (Sotomayor y Ramírez, 2014). Igualmente, esta especie presenta índices materiales (Ashby, 2005), que relacionan sus módulos de elasticidad con su densidad, mayores en comparación con maderas de densidades más altas (Sotomayor, Reyes, Rincón y Suárez, 2013). Es decir, la madera de G. americanus es porosa pero resistente. La combinación de estos dos aspectos sugiere que esta madera se compacta más fácilmente con respecto a su capacidad para comprimirse.
En el mismo contexto, el coeficiente de variación del coeficiente de densificado fue $255 \%$ mayor con respecto al coeficiente de compresión. Es decir, la compresión de la madera fue más uniforme en comparación con el nivel de densificado correspondiente. Con todo, el coeficiente de compresión de la madera de G. americanus se situó al interior del intervalo de $40 \%$ a $65 \%$, valores reportados entre otros investigadores por Sandberg y Navi (2007), Yoshihara y Tsunematsu (2007), Fang et al. (2012b) y Khalil et al. (2014).

\section{Densidad}

Después del tratamiento la densidad de G. americanus aumentó $102 \%$ (tabla 1 ). Este resultado es comparable con los de trabajos anteriores de Yoshihara y Tsunematsu (2007), que encontraron para madera de Picea sitchensis con densidad de $480 \mathrm{~kg}$ $\mathrm{m}^{-3}$, un incremento en su densidad de $90 \%$. Por su parte Fang, Cloutier, Blanchet y Koubaa (2012a), para madera de Populus tremuloides después del densificado su densidad aumentó de $348 \mathrm{~kg} \mathrm{~m}^{-3}$ a $687 \mathrm{~kg} \mathrm{~m}^{-3}$; es decir, un 97.4\%. Igualmente, Haller y Wehsener (2004) al densificar madera de Picea abies, con densidad de $390 \mathrm{~kg} \mathrm{~m}^{-3}$, reportan un incremento en la densidad de $123 \%$ después de densificar la madera. De la misma manera, en el caso de Kamke (2006), con Pinus radiata de densidad de $412 \mathrm{~kg} \mathrm{~m}^{-3}$, se reportó un incremento en la densidad de la madera densificada de $78 \%$.

Los datos de la tabla 1 pueden ser comparados con los índices de densificado mostrados en la tabla 2, que indican el incremento en la densidad de la madera ocasionado por su densificado. En esta tabla los índices de densificado y de calidad del densificado para G. americanus son los mismos reportados por Sotomayor (2017). En efecto, los tratamientos de densificado aplicados por Kutnar et al. (2008), Skyba et al. (2009) y Ulker, Imirzi y Burdurlu (2012) mejoraron sensiblemente la densidad de las maderas estudiadas. Aún más el índice de calidad de densificado $(Q)$ determinado para $G$. americanus fue mayor al de los autores citados. 
Tabla 2. Índice de densificado, índice elástico e índice de calidad de densificado para madera densificada de G. americanus y resultados de la bibliografía.

\begin{tabular}{|c|c|c|c|}
\hline \multicolumn{4}{|c|}{ G. americanus } \\
\hline & $l_{\rho}$ & $I M O E$ & $Q$ \\
\hline $\bar{x}$ & $2.01 *$ & 2.15 & $0.95^{*}$ \\
\hline$\sigma$ & 0.08 & 0.21 & 0.09 \\
\hline \multicolumn{4}{|c|}{$\begin{array}{c}3.90 \\
\text { Populus deltoides x Populus trichocarpa }\end{array}$} \\
\hline Kutnar et al. (2008) & $\begin{array}{c}1.66 \\
\text { Picea abies }\end{array}$ & 1.38 & 0.83 \\
\hline Skyba et al. (2009) & $\begin{array}{c}3.52 \\
\text { Fagus sylvatica }\end{array}$ & 2.29 & 0.65 \\
\hline Skyba et al. (2009) & $\begin{array}{c}1.75 \\
\text { Pinus sylvestris }\end{array}$ & 1.19 & 0.68 \\
\hline Ulker et al. (2012) & 1.84 & 1.11 & 0.61 \\
\hline Promedio referencias & 2.19 & 1.49 & 0.69 \\
\hline
\end{tabular}

$\mathrm{I}_{\rho}=$ Índice de densificado; $\mathrm{I}_{\mathrm{MOE}}=$ Índice elástico; $\mathrm{Q}$ = Índice de calidad del densificado; * Sotomayor (2017).

Fuente: elaboración propia del autor.

\section{Módulo de elasticidad}

Después del densificado, el módulo de elasticidad de la madera de G. americanus, se incrementó en $113 \%$ (tabla 1), mientras que el módulo dinámico en la dirección longitudinal y determinado con ondas de esfuerzo, reportado por Sotomayor (2016), se incrementó en $90.5 \%$. Como consecuencia de la diferencia en las configuraciones experimentales, estos resultados son difíciles de comparar. Sin embargo, la magnitud del incremento en la densidad y en el módulo dinámico de G. americanus es comparable con los resultados reportados entre otros investigadores por Haller y Wehsener (2004), que densificaron madera de Picea abies, con densidad de $390 \mathrm{~kg} \mathrm{~m}^{-3}$ y reportan un incremento en el módulo de elasticidad de 62\%; Kamke (2006) que densificó Pinus radiata con densidad de $412 \mathrm{~kg} \mathrm{~m}^{-3}$ y obtuvo un incremento en el módulo de elasticidad de $116 \%$ y Yoshihara y Tsunematsu (2007) quienes densificaron madera de Picea sitchensis con densidad de $480 \mathrm{~kg} \mathrm{~m}^{-3}$ consiguen que el módulo de elasticidad incremente en $70 \%$.
Por su parte, Kutnar et al. (2008) obtienen para madera densificada $\left(\lambda_{\rho}=63 \%\right)$ de Populus deltoides $\mathrm{x}$ Populus trichocarpa un incremento en el módulo de elasticidad en flexión estática de 37\%. En el mismo contexto, Gao et al. (2016) consiguen para madera densificada de Populus tomentosa $\left(490 \mathrm{~kg} / \mathrm{m}^{3}<\rho_{\mathrm{CH}}<588 \mathrm{~kg} / \mathrm{m}^{3}\right)$ con un coeficiente de densificado de $47 \%$, obtuvieron un incremento en el módulo de elasticidad en flexión estática de $73.2 \%$.

\section{Evaluación del tratamiento de densificado}

La temperatura del tratamiento fue menor que la temperatura de suavizado de los principales polímeros constituyentes de la madera: celulosa 200 ${ }^{\circ} \mathrm{C}$ a $250{ }^{\circ} \mathrm{C}$, hemicelulosas $150{ }^{\circ} \mathrm{C}$ a $220{ }^{\circ} \mathrm{C}$ y lignina $210^{\circ} \mathrm{C}$ (Sandberg y Navi, 2007). No obstante, la madera en tanto que material viscoelástico (Kutnar et al., 2008), durante la etapa de suavizado, el contenido de humedad de la madera, que fue superior al punto de saturación de la fibra, combinado con la temperatura de $93.5{ }^{\circ} \mathrm{C}$, plastificaron 
la madera y facilitaron su densificado. Por el contrario, durante el tiempo de secado y la reducción del contenido de humedad a $9.7 \%$, se liberaron los esfuerzos de compresión, manteniendo el coeficiente de compresión constante gracias al dispositivo de densificado. Esta estrategia experimental permitió que la madera se deformara con un deterioro mínimo, lo que posiblemente permitió el fenómeno de densificación acompañado del aumento del módulo de elasticidad proporcionalmente al incremento en la densidad de la madera de G. americanus (Laine et al., 2013).

Este resultado se presenta en la figura 1, donde se muestran para los resultados antes y después del densificado, el módulo de elasticidad en función de la densidad, sus regresiones y sus coeficientes de determinación. Antes del densificado, la dependencia del módulo de elasticidad en función de la densidad se explica con un coeficiente de determinación medio y la densidad se reparte en un intervalo de $328 \mathrm{~kg} \mathrm{~m}^{-3}$ a $447 \mathrm{~kg} \mathrm{~m}^{3}$. En cambio, después del densificado la relación entre el módulo de elasticidad y la densidad se incrementa, y registra un fuerte coeficiente de determinación. En cambio, el rango de valores de la densidad se amplía en un intervalo más grande que va desde 604 $\mathrm{kg} \mathrm{m}^{-3}$ a $890 \mathrm{~kg} \mathrm{~m}^{-3}$. Cuantitativamente, la ganancia en la densidad de $101.4 \%$, correspondió un incremento en el módulo de elasticidad de $114.7 \%$. De aquí se desprende que la baja intensidad de las variables del tratamiento de densificado higrotermo-mecánico aseguraron, hasta cierto punto, la homogeneidad y la repetividad en las características de la madera de G. americanus.

\section{Conclusiones}

El aumento artificial de la densidad de la madera de G. americanus incrementa su densidad y su módulo de elasticidad.

Los índices de densificado, elástico y potencial, confirmaron que el tratamiento de densificado higro-termo-mecánico produjo buenos resultados para madera de baja densidad.

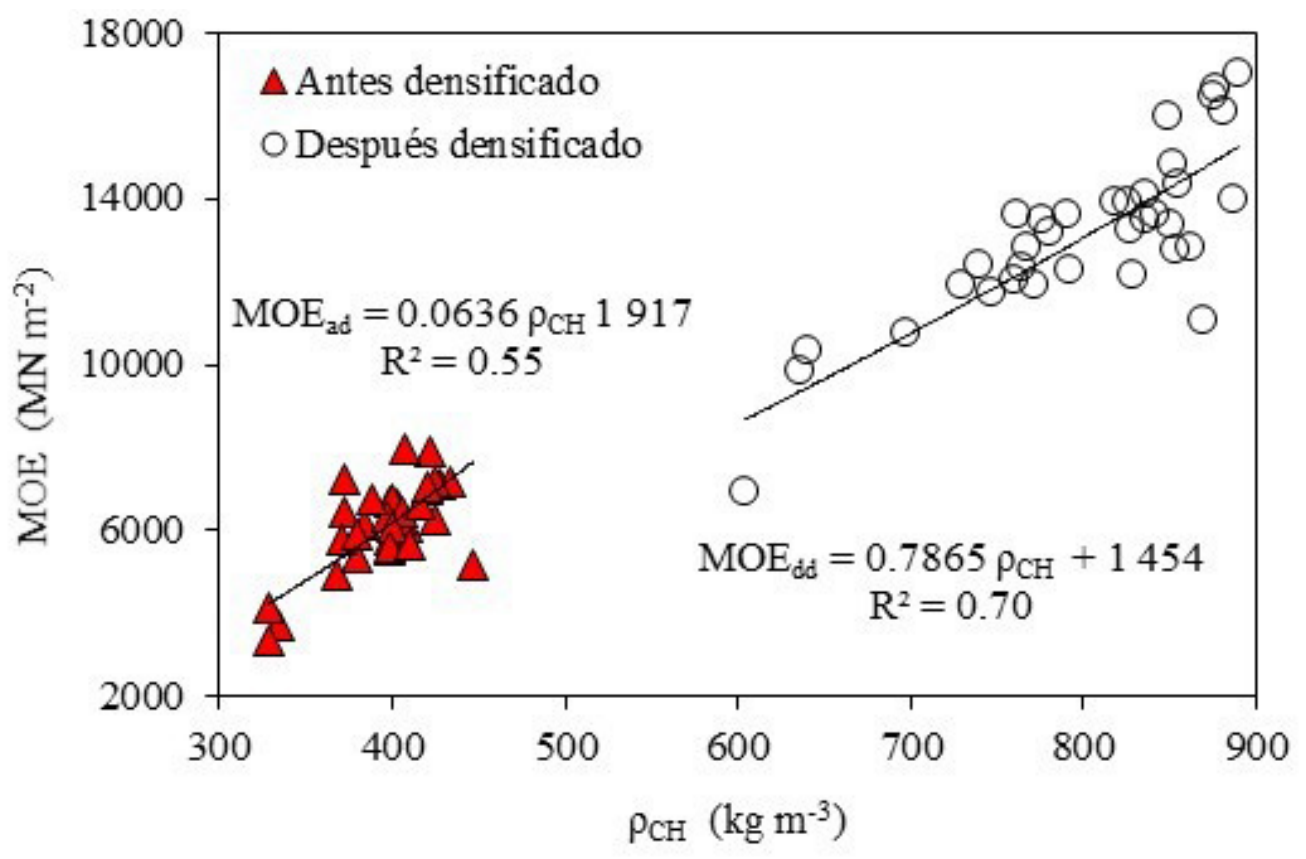

Figura 1. Módulo de elasticidad (MOE) en función de la densidad $\left(\rho_{\mathrm{CH}}\right)$, sus regresiones y sus coeficientes de determinación $\left(\mathrm{R}^{2}\right)$.

Fuente: elaboración propia del autor. 
El tratamiento de densificado higro-termo-mecánico puede transformar madera de baja densidad en un nuevo material con un mayor módulo de elasticidad. De esta forma, el densificado puede valorizar especies que por su baja densidad son desfavorecidas para fines de diseño de productos y estructuras de madera.

Se recomienda extender esta línea de investigaciones considerando el efecto del densificado en la composición química de la madera y en su estructura anatómica.

\section{Referencias}

Aimene, Y. E. y Nairn, J. A. (2015). Simulation of transverse wood compression using a large-deformation, hyperelastic-plastic material model. Wood Science and Technology, 49(1), 21-39. DOI: https://doi.org/10.1007/ s00226-014-0676-6

Asaba, M. y Nishimura, H. (2001). Effect of manufacturing conditions on bending strength of compressed wood. Transactions of the Japan Society of Mechanical Engineers, Part A, 67(654), 267-272. DOI: https://doi. org/10.1299/kikaia.67.267

Ashby, M. F. (2005). Materials selection in mechanical design. Oxford: Butterworth Heinemann.

Blomberg, J., Persson, B. y Blomberg, A. (2005). Effects of semi-isostatic densification of wood on the variation in strength properties with density. Wood Science and Technology, 39(5), 339-350. DOI: https://doi.org/10.1007/ s00226-005-0290-8

Cordero, J. y Boshier, D. H. (2003). Árboles de Centroamérica. Turrialba: Centro Agronómico Tropical de Investigación y Enseñanza.

Dinwoodie, M. (2002). Timber: its nature and behavior. Nueva York: CRC Press.

Eckelman, C. A. (1993). Potential uses of laminated veneer lumber in furniture. Forest Products Journal, 43(4), 19-24. Recuperado de https:// www.agriculture.purdue.edu/fnr/faculty/eckel$\underline{\text { man/documents/d199304b.pdf }}$
Erdil, Y. Z., Kasal, A., Zhang, J. L., Efe, H. y Dizel, T. (2009). Comparison of mechanical properties of solid wood and laminated veneer lumber fabricated from Turkish beech. Forest Products Journal, 59(6), 55-60.

Fang, C. H., Cloutier, A., Blanchet, P., Koubaa, A. y Mariotti, N. (2011). Densification of wood veneers combined with oil-heat treatment. Part I: Dimensional stability. BioResources, 6(1), 373-385. Recuperado de http://ojs.cnr. ncsu.edu/index.php/BioRes/article/view/BioRes $06 \quad 10373$ Fang_CBK M Densification Wood Veneers Oil Heat/818

Fang, C. H., Cloutier, A., Blanchet, P. y Koubaa, A. (2012a). Densification of wood veneers combined with oil heat treatment. Part II: hygroscopicity and mechanical properties. BioResources, 7(1), 925-935. Recuperado de http://ojs.cnr.ncsu.edu/index.php/BioRes/article/view/BioRes 0710925 Rang_CBK Densification Wood Veneers Oil Heat/1363

Fang, C. H., Mariotti, N., Cloutier, A., Koubaa, A. y Blanchet, P. (2012b). Densification of wood veneers by compression combined with heat and steam. European Journal of Wood and Wood Products, 70(1), 155-163. DOI: https:// doi.org/10.1007/s00107-011-0524-4

Gao, Z., Huang, R., Lu, J., Chen, Z., Guo, F. y Zhan, T. (2016). Sandwich compression of wood: control of creating density gradient on lumber thickness and properties of compressed wood. Wood Science and Technology, 50(4), 833-844. DOI: https://doi.org/10.1007/ s00226-016-0824-2

Gutiérrez P., H. y de la Vara S., R. (2011). Análisis y diseño de experimentos. México: McGraw Hill.

Haller, P. y Wehsener, J. (2004). Mechanical properties of densified spruce. Holz Als Roh-Und Werkstoff, 62(6), 452-454. DOI: https://doi. org/10.1007/s00107-0040516-8

Hayashi, S. y Nishimura, H. (2001). Study on static \& dynamic bending strength of compressed lumber and the utilization for reinforcement. Transactions of the Japan Society of Mechanical 
Engineers, Part A. 67(656), 757-762. DOI: https://doi.org/10.1299/kikaia.67.757

Hayashi, T. y Oshiumi, S. (1993). Bending strength distribution of laminated veneer lumber for structural use. Mokuzai Gakkaishi, 39(9), 985-992.

Hill, C. A. S. (2006). Wood modification: chemical, thermal and other processes. Hoboken: Wiley. DOI: https://doi.org/10.1002/0470021748

International Organization for Standardization (2012). ISO 3129:2012. Wood. Sampling methods and general requirements for physical and mechanical tests. Bruselas: International Organization for Standardization.

International Organization for Standardization (2014). ISO 13061-1:2014. Physical and mechanical properties of wood. Test methods for small clear wood specimens. Part 1: Determination of moisture content for physical and mechanical tests. Bruselas: International Organization for Standardization.

Kamke, F. A. (2006). Densified radiata pine for structural composites. Maderas: Ciencia y Tecnología, 8(2), 83-92. DOI: https://doi. org/10.4067/S0718221X2006000200002

Khalil, H. P. S., Dungani, R., Issam, A. M., Hossain Md. S., SriAprilia N. A., Budiarso A. y Rosamah E. (2014). Determination of the Combined Effect of Chemical Modification and Compression of Agatis Wood on the Dimensional Stability, Termite Resistance, and Morphological Structure. Bioresources, 9(4), 6614-6626. DOI: https://doi.org/10.15376/ biores.9.4.6614-6626

Koman, S., Feher, S., Abraham, J. y Taschner, R. (2013). Effect of knots on the bending strength and the modulus of elasticity of wood. Wood Research, 58(4), 617-626. Recuperado de https://www.researchgate.net/ publication/289961696 Effect of knots on the bending strength and the modulus of elasticity of wood

Kubojima, Y., Ohtani, T. y Yoshihara, H. (2004). Effect of shear deflection on bending properties of compressed wood. Wood and Fiber Science, 36(2), 210-215. Recuperado de https://wfs.swst.org/index.php/wfs/article/ view/1316/1316

Kutnar, A., Kamke, F. A. y Sernek, M. (2008). The mechanical properties of densified VTC wood relevant for structural composites. Holz als Roh- und Werkstoff, 66(6), 439-446. DOI: https://doi.org/10.1007/ s00107-008-0259-z

Laine, K., Belt, T., Rautkari, L., Ramsay, J., Hill, C. A. S. y Hughes M. (2013). Measuring the thickness swelling and set-recovery of densified and thermally modified Scots pine solid wood. Journal of Materials Science, 48(24), 8530-8538. DOI: https://doi.org/10.1007/ s10853-013-7671-4

Navi, P. y Pizzi, A. (2015). Property changes in thermo-hydro-mechanical processing. Holzforschung, 69(7), 863-873. DOI: https://doi. org/10.1515/hf-2014-0198

Navi, P. y Girardet, F. (2000). Effects of thermo-hydro-mechanical treatment on the structure and properties of wood. Holzforschung, 54(3), 287-293. DOI: https://doi.org/10.1515/ HF.2000.048

Niklas, K. J. y Spatz, H. C. (2010). Worldwide correlations of mechanical properties and green wood density. American Journal of Botany, 97(10), 1587-1594. DOI: https://doi. org/10.3732/ajb.1000150

Ozarska, B. (1999). A review of the utilization of hardwoods for LVL. Wood Science and Technology, 33(4), 341-351. DOI: https://doi. org/10.1007/s002260050120

Rivera, J. E. (2006). Notas sobre Hernandiaceae: primer registro de Gyrocarpus americanus Jacq. para México y de Sparattanthelium amazonum Mart. para Oaxaca. Acta Botánica Mexicana, 78, 67-76. DOI: https://doi. org/10.21829/abm78.2007.1030

Sandberg, D. y Navi, P. (2007). Introduction to Thermo-hydro-mechanical (THM) Wood Processing. Växjö: Växjö University. 
Skyba, O., Schwarze, F. y Niemz, P. (2009). Physical and Mechanical Properties of Thermo-Hygro-Mechanically (THM)-Densified Wood. Wood Research, 54(2), 1-18. Recuperado de https://www.researchgate.net/ publication/289633986 Physical and mechanical proper ties of Thermo-hygromechanically THM - Densified wood

Sotomayor C., J. R. (2015). Banco FITECMA de características físicomecánicas de maderas mexicanas. Morelia: Universidad Michoacana de San Nicolás de Hidalgo. Recuperado de https://www.researchgate.net/profile/Javier Ramon Sotomayor Castellanos2/publicati on/276841418 Banco FITECMA de caracteristicas fisicomecanicas de maderas Mexicanas/links/555a2dc408aeaaff3bfabb2c/ BancoFITECMA-de-caracteristicas-fisico-mecanicas-de-maderas-Mexicanas.pdf

Sotomayor, J. R. (2016). Efecto del densificado de la madera de Gyrocarpus americanus Jacq. en su módulo dinámico determinado por ondas de esfuerzo. Ciencia amazónica (Iquitos), 6(2), 162-171. DOI: https://doi.org/10.22386/ ca.v6i2.117

Sotomayor, J. R. (2017). Densificado higro-termo-mecánico de madera de Gyrocarpus americanus. Evaluación por ultrasonido. Revista de Investigaciones Agropecuarias, 43(2), 156164. Recuperado de https://www.academia. edu/34403140/Densificado higro-termomec\%C3\%A1nico de madera de Gyrocarpus americanus. Evaluaci\%C3\%B3n por ultrasonido

Sotomayor, J. R. y Villaseñor A, J. M. (2016). Coeficientes de higroelasticidad en flexión estática de la madera de Pinus douglasiana procedente de Michoacán. Investigación e Ingeniería de la Madera, 12(1), 36-51. Recuperado de $\quad$ https://www.academia.edu/24919116/
Coeficientes de higroelasticidad en flexi\%C3\%B3n est $\%$ C3\%A1tica de la madera de Pinus douglasiana procedente de $\mathrm{Mi}$ choac $\%$ C3\%A1n

Sotomayor, J. R. y Ramírez P., M. (2014). Características físicas de 12 maderas mexicanas. Investigación e Ingeniería de la Madera, 10(1), 4-35. Recuperado de https://www.researchgate.net/publication/269095842 Caracteristicas fisicas de 12 ma deras mexicanas

Sotomayor, J. R., Reyes R., L. A., Rincón G., E. U. y Suárez B., G. (2013). Módulos de elasticidad dinámicos e indicadores de calidad de cinco maderas mexicanas estudiadas por métodos no destructivos. Investigación e Ingeniería de la Madera, 9(1), 3-20. Recuperado de https://www.researchgate.net/ publication/261064731 Investigacion_e Ingenieria de la Madera 2013 Vol 9 No 1

Sotomayor, J. R., Ramírez P., M. y Olguín C., J. B. (2014). Caracterización en flexión estática de madera plastificada de Quercus scytophylla. Ciencia Nicolaíta, 62,17-33. Recuperado de https://www.cic.cn.umich.mx/index.php/cn/ article/view/192

Tang, X., Nakao T. y Zhao, G. (2004). Physical properties of compressed wood fixed via different heating pathways to obtain a constant recovery. Mokuzai Gakkaishi, 50(5), 333-340.

Ulker, O., Imirzi, O. y Burdurlu, E. (2012). The Effect of Densification Temperature on Some Physical and Mechanical Properties of Scots Pine (Pinus sylvestris L.). BioResources, 7(4), 5581-5592. DOI: https://doi.org/10.15376/ biores.7.4.5581-5592

Yoshihara, H. y Tsunematsu, S. (2007). Bending and shear properties of compressed Sitka spruce. Wood Science and Technology, 41(2), 117-131. DOI: https://doi.org/10.1007/ $\underline{\text { s00226-006-0091-8 }}$ 